\title{
Neural Circuits Mediating Visual Flight Control in Flies. I. Quantitative Comparison of Neural and Behavioral Response Characteristics
}

\author{
Klaus Hausen a and Christian Wehrhahn \\ Max-Planck-Institut für biologische Kybernetik, D-7400 Tübingen, FRG
}

The motion-sensitive horizontal cells in the lobula plate of the fly are assumed to play a key role in the sensory control of yaw torque generated by the flying animal during coursestabilization maneuvers and the fixation of objects. This inference results from comparisons of electrophysiological data obtained from blowflies (Calliphora erythrocephala) and behavioral data obtained mainly from houseflies (Musca domestica) and fruitflies (Drosophila melanogaster). Apart from few exceptions, the compatibility of these physiological and behavioral data has not been critically tested. In the present study, the responses of the equatorial horizontal cell HSE of Calliphora and the yaw torque responses of Calliphora and Musca were recorded under identical visual stimulation with moving periodic gratings. The goal of the experiments was to obtain electrophysiological and behavioral data on Calliphora, on the one hand, and behavioral data on Calliphora and Musca, on the other hand, that allow direct comparisons between the physiological properties of the HSE and the visually induced torque responses in both species.

The dependence of the HSE responses and the yaw torque responses on the direction, contrast frequency, and brightness of a moving periodic grating were evaluated quantitatively. The results of the electrophysiological recordings and torque measurements are in close agreement and thus represent strong evidence that the horizontal cells are, in fact, involved in yaw torque control in both species. Measurements of the cellular and behavioral responses as function of the stimulus position in the visual field, however, reveal differences between the spatial sensitivity of the horizontal cells and the sensory input to the motor system. This suggests the existence of an additional torque control system. The roles of the horizontal cells and of the additional system in the visual orientation behavior of the fly are discussed.

Visual orientation in animals and humans relies largely on the analysis of retinal motion patterns by the visual system. These patterns contain information on self-motion, object motion, and

\footnotetext{
Received Feb. 21, 1989; revised Apr. 19, 1989; accepted Apr. 28, 1989.

We would like to thank T. Wiegand and H. Kümmerle for expert technical assistance during the experiments and for drawing the figures, and I. Geiss for typing the manuscript. Helen Brew, John Miller, and the referees are thanked for numerous helpful comments.

Correspondence should be addressed to Christian Wehrhahn, Max-Planck-Institut für biologische Kybernetik, Spemannstrasse 38, D-7400 Tübingen, West Germany.

a Present address: Zoologisches Institut der Universität, Weyertal 119, D-5000 Köln 41, West Germany.

Copyright (C) 1989 Society for Neuroscience $0270-6474 / 89 / 113828-09 \$ 02.00 / 0$
}

the three-dimensional structure of the surroundings, which can be used for the orientation in complex environments.

Course stabilization and object fixation are two major components of visually guided behavior that have been studied extensively using flies as a model system (Götz, 1983; Heisenberg and Wolf, 1984; Wehrhahn, 1985; Reichardt, 1986; Egelhaaf et al., 1988). Visual course stabilization relies on the evaluation of global retinal motion patterns perceived by the animal during self-motion in a stationary environment. For example, apparent rotations of the entire surroundings, which indicate deviations from a straight course, induce corrective flight torque responses around the body axes. In contrast, fixation behavior is characterized by turnings of the flying animal toward stationary or moving objects, as can be observed during approach to landing sites or in tracking sequences between two flies. Studies of the mechanism underlying fixation have caused controversy, but it is generally accepted now that fixation also relies on the evaluation of motion (Reichardt, 1973; Pick, 1976; Wehrhahn and Hausen, 1980). Recent studies demonstrate that, in particular, the computation of relative motion between object and background plays an important role (Poggio et al., 1981; Reichardt et al., 1983; Egelhaaf, 1985a-c).

Analysis of the visual system of the fly has revealed that the main motion computation center of the optic lobe is located in the lobula plate. This neuropil contains an array of about 50 large directionally selective interneurons that encode retinal motion patterns and are considered to play a key role in visual orientation behavior (Hausen, 1981, 1984; Hausen and Egelhaaf, 1988; Egelhaaf et al., 1988). Major output neurons of the lobula plate are 3 giant cells, which respond selectively to horizontal motion. The cells are termed "northern," "equatorial," and "southern" horizontal cell (HSN, HSE, HSS) and scan the dorsal, medial, and ventral part of the ipsilateral visual field, respectively. They are synaptically coupled to descending neurons that converge, together with other sensory pathways, onto the motor control centers in the thoracic ganglion (Hausen, 1976, 1982a, b; Eckert, 1981; Heide, 1983; Strausfeld and Bassemir, 1985; Hausen and Hengstenberg, 1987; Hausen et al., 1988; Hengstenberg et al., 1988; K. Hausen, unpublished observations). Further output elements of the lobula plate are four recently described figure-detection cells (FD cells) that, in contrast to the horizontal cells, respond selectively to horizontal movement of small objects in the visual environment (Egelhaaf, $1985 \mathrm{~b}, \mathrm{c})$. Although the synaptic connections of these elements have not yet been investigated, they are also assumed to be coupled via descending neurons to the thoracic motor centers.

Several studies performed with different experimental techniques and in different species of flies indicate that motioninduced yaw torque responses of these animals are controlled 
by 2 neural systems evaluating large field and object motion and that the HS and FD neurons are the essential sensory output elements of these systems (Heisenberg et al., 1978; Geiger and Nässel, 1982; Hausen and Wehrhahn, 1983; Reichardt et al., 1983; Egelhaaf, 1985a, b, c; Bausenwein et al., 1986). This inference critically depends on the assumption that physiological and behavioral data obtained from different species are compatible, since the electrophysiological data available at present were obtained from studies in blowflies (Calliphora erythrocephala), whereas the bulk of the behavioral data results from investigations in houseflies (Musca domestica) and fruitflies (Drosophila melanogaster). Comparative anatomical investigations demonstrate that at least the horizontal cells in various species of flies, including Musca and Drosophila, are very similar to those found in blowflies. This structural conformity suggests similar physiological properties of the cells in these species (Hausen, unpublished observations).

The present study concerns the role of the HS cells in the control of motion-induced flight torque responses in flies and has 2 aims. On the one hand, cellular and behavioral responses to motion are measured under identical stimulation conditions in Calliphora to obtain comparable physiological and behavioral data in this species. On the other hand, the torque responses of Calliphora and Musca are measured under the same conditions to obtain reliable data for interspecific comparisons.

In a subsequent study (Hausen and Wehrhahn, 1990), the functional significance of the HS- and FD-cells in torque control is further investigated by means of microsurgical lesion experiments.

\section{Materials and Methods}

Animals. Behavioral experiments were carried out with female houseflies (Musca domestica) and blowflies (Calliphora erythrocephala). The electrophysiological recordings were performed exclusively on female blowflies. The flies were reared in a laboratory culture and were aged 2-18 d.

Stimulation. The animals were stimulated with a moving periodic grating of bright and dark stripes. The grating had an angular diameter $\mathrm{d}=39.6^{\circ}$, a spatial wavelength $\lambda=13.2^{\circ}$, a contrast $\mathrm{c}=0.8$, and a mean luminance $I=70 \mathrm{~cd} \cdot \mathrm{m}^{-2}$. The standard position of the grating was in the right visual field of the test flies at $\psi=+40^{\circ}$ and $\theta=0^{\circ}$. $\psi$ and $\theta$ denote the azimuth and elevation angles in the visual field of the fly and are defined such that they are positive in the right and in the dorsal hemisphere, respectively. The periodic grating and its direction of motion could be tilted by an angle $\alpha$ (see inset of Fig. $2 a$ ): $\alpha=0^{\circ}, 180^{\circ}$ : horizontal backward, forward motion; $\alpha=90^{\circ}, 270^{\circ}$ : vertical downward, upward motion. Pattern velocities were specified in terms of the contrast frequency $\omega / \lambda$ ( $\omega$ is angular velocity and $\lambda$ spatial wavelength of the grating). Standard pattern direction and contrast frequency in the experiments were $\alpha=0^{\circ}$ and $\omega / \lambda=1 \mathrm{~Hz}$. In some experiments, a second, stationary grating was placed in the left visual field of the flies at $\psi=$ $-45^{\circ}, \theta=0^{\circ}$. Stimulation was performed according to the standard program, in which grating-motion was repetitively displayed for periods of $1.5 \mathrm{sec}$ in intervals of $9.3 \mathrm{sec}$.

Behavioral experiments. Flies were prepared for behavioral measurements by glueing a small cardboard triangle to their tergites, which served to attach them to a torque meter. The heads of the flies were fixed with wax to the thorax to prevent head movements. The occlli and, in monocular stimulation experiments, also the left compound eyes were covered with black nontoxic casein paint. The preparation was performed under light anesthesia with carbon dioxide, and the animals were allowed to recover from it for at least one night before experiments started.

In the experiments, the flies were suspended from a torque meter (Fermi and Reichardt, 1963) and carefully adjusted with respect to the grating. Position and orientation of the animals were controlled by microscopic inspection of the frontal equatorial pseudopupils of the compound eyes, which indicate precisely the head orientation in space.
Stimulation was performed according to the standard program, and the stimulus-induced flight torque signals of the tethered flying animals were recorded, digitized, and stored for later evaluation.

If not otherwise stated, the behavioral data presented in the study arc averages of a total of 20 measurements carried out with 12 animals under each stimulus condition. Positive and negative values of the torque data denote intended turnings of the flying animals to the right and left, respectively.

Electrophysiological experiments. Animals were briefly anesthetized and glued onto a small preparation platform. The ocelli were covered with black paint. In the case of monocular stimulation experiments, the left compound eye was also painted black. A small hole was cut above the right lobula plate into the rearside of the head capsula for insertion of the recording electrode. The legs, the proboscis and its retractor muscles, and the esophagus were dissected off in order to reduce movements of the brain during recording. The animals were then positioned in the experimental setup and oriented with respect to the grating under microscopic control as described previously. During the experiments, the animals were supplied with saline and oxygen (see Hausen, 1982a, for further details).

All measurements were performed on the HSE of the right lobula plate. The graded responses of the cell to the motion stimuli presented in the standard program were recorded intracellularly and stored on magnetic tape for later analysis. The recordings were performed with microelectrodes filled with 3\% (wt/vol) aqueous solution of the fluorescent dye Lucifer yellow CH (Stewart, 1978), and the identity of each recorded cell was checked after the experiment by iontophoretic dye injection and subsequent inspection of the brain under the fluorescence microscope.

Except for the transient recordings shown in Figure 1, the electrophysiological data presented in this study are response averages based on 15 measurements performed with 5 animals.

\section{Results}

Transient cellular and behavioral responses under stimulation with horizontal motion

In 2 initial series of experiments, the transient responses of the HSE and the transient flight torque responses of blowflies to horizontal motion were recorded. The flies were stimulated in these experiments with 2 gratings placed symmetrically in front of the left and right eye at $\psi= \pm 45^{\circ}, \theta=0^{\circ}$. The left grating was kept stationary; the right grating was moved horizontally with a contrast frequency of $\omega / \lambda=1 \mathrm{~Hz}$. The motion stimuli were displayed according to the standard program. The graded signals of the HSE and the flight torque signals of the fixed flying flies were continuously recorded and averaged. The resulting response patterns are shown in Figure 1.

The signal trace of the HSE (Fig. $1 a$ ) shows that the cell is a directionally selective interneuron responding to horizontal motion. Stimulation with back to front motion leads to a hyperpolarization (response amplitude $\mathrm{H}=-4.6 \mathrm{mV}$ ), whereas front to back motion induces a massive depolarization $(\mathrm{H}=+8.6$ $\mathrm{mV}$ ) of the cell with respect to the resting potential $(-48 \mathrm{mV})$. The responses are brisk and sustained, showing only a weak adaptation during the stimulation interval. The flight torque responses of the blowfly to these motion stimuli are shown in Figure $1 b$. Stimulation with front to back motion leads to a significant positive flight torque signal $(\mathrm{T}=+7$ dyne $\mathrm{cm})$, indicating an intended turning with respect to the grating motion, whereas stimulation with back to front motion is ineffective. Further measurements (see below) demonstrate that the responses to back to front motion are rather variable in Calliphora and that weak responses of opposite sign also can be elicited under this condition. A comparison between the 2 recordings shows that onset and decay of the torque response to motion are slow as compared to the respective signal components in the HSE. 


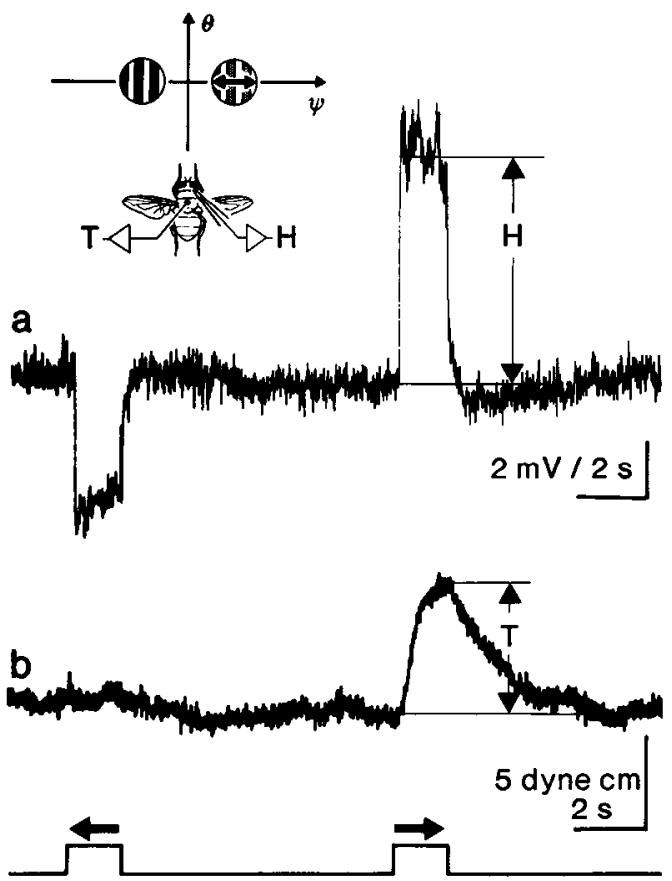

Figure 1. Transient graded responses of the equatorial horizontal cell HSE $(a)$ and transient torque responses of tethered flying flies $(b)$ to stimulation with motion. Both rccordings were performed with Calliphora under identical stimulation conditions, with two periodic gratings placed at $\psi= \pm 45^{\circ}, \theta=0^{\circ}$ in the frontal visual field of the fly (inset). Each grating had a diameter $\mathrm{d}=39.6^{\circ}$, a spatial wavelength $\lambda=13.2^{\circ}$, and a mean luminance $\mathrm{I}=70 \mathrm{~cd} \mathrm{~m}^{-2}$. The right grating was moved horizontally with a contrast frequency of $1 \mathrm{~Hz}$. The left grating was kept stationary. The motion stimuli are indicated at the bottom of the figure. The response traces shown are averages of 150 intracellular measurements in 5 HSE of different animals and of 200 behavioral measurements in 14 flies. The HSE responds with a graded hyperpolarization (negative signal deflection) and depolarization (positive deflection) to stimulation with back to front and front to back motion, indicating that the cell is activated by front to back motion. Positive flight torque responses, indicating intended clockwise turnings of the fixed flying animals, are elicited under stimulation with front to back motion. The response amplitudes $H$ and $T$ are evaluated quantitatively in the following experiments.

The transient flight torque responses of Musca to this stimulation (not shown in Fig. 1) resemble closely those of Calliphora. They differ from the illustrated signal trace only with respect to the response to back to front motion, which can always be observed in $\mathrm{Musca}$, and the time course of the response after the end of the motion stimuli (Hausen and Wehrhahn, 1990).

Hence, monocular stimulation with front to back motion activates the ipsilateral HSE and simultaneously induces a strong torque response of the flying animal following the direction of motion. The results are in full accordance with previous electrophysiological measurements in Calliphora (Hausen, 1982b) and torque measurements in Calliphora and Musca (Hausen and Wehrhahn, 1983; Wehrhahn, 1987). The dependence of the cellular and behavioral responses on various stimulus parameters are evaluated quantitatively in the experiments described next.

\section{Response dependence on the direction of motion}

In this experimental series, the responses of the HSE of Calliphora and the flight torque responses of Musca and Calliphora were evaluated as functions of the direction of the grating mo- tion. The grating was positioned in the right visual field at $\psi=$ $+40^{\circ}, \theta=0^{\circ}$, and its orientation and direction of motion were changed in steps of $30^{\circ}$. In each orientation, grating motion was displayed according to the standard program. The left eyes of the tested flies were painted black in these and all following experiments in order to exclude response contributions from the left optic lobe. The results of the experiments are shown in Figure 2.

The electrophysiological data (Fig. 2a) illustrate the directional characteristics of the HSE. The cell responds with maximal depolarization to stimulation with front to back motion $\left(\alpha=0^{\circ}\right.$; see inset) and is maximally inhibited by motion in the reverse direction. The response amplitudes decrease when the direction of motions deviates from the preferred direction. The curve approximates cosine $(\alpha)$, which means that the HSE is driven by the horizontal projection of the motion vector.

The behavioral measurements yield similar results (Fig. $2 b$ ). In both species, the induced flight torque responses reach peak values under stimulation with front to back motion and decline gradually when the direction of motion is changed. Negative torque responses to back to front motion are weak and clearly expressed only in Musca.

Thus, the response dependencies on the direction of motion found in the electrophysiological and behavioral experiments on Calliphora are highly similar. A comparison of the torque responses of both species of flies shows that they are also in close agreement with each other except for the difference in amplitude under stimulation with back to front motion. The latter is regarded as a minor quantitative discrepancy rather than an indication of a qualitative difference in the sensory input organization of the motor systems in the 2 species.

\section{Response dependence on the contrast frequency of motion}

The cellular responses of the HSE in Calliphora and the flight torque responses of Calliphora and $\mathrm{Musca}$ were evaluated using the same experimental conditions as described in the last section except that the direction of motion was kept constant at $\alpha=0^{\circ}$ and that the contrast frequency $\omega / \lambda$ of the grating was changed in discrete steps. The results of the experiments are compiled in Figure 3.

The electrophysiological measurements (Fig. $3 a$ ) reveal that the HSE responds to a wide range of contrast frequencies. The lower and upper response thresholds of the cell lie at about 0.005 $\mathrm{Hz}$ and $50 \mathrm{~Hz}$, respectively, and the response maximum is reached at contrast frequencies of $1-5 \mathrm{~Hz}$. The results of the respective torque measurements in Musca and Calliphora (Fig. $3 b$ ) reveal some interspecies differences but are consistent with the electrophysiological data. In both species of flies, the lower and upper response thresholds lie at about $0.005-0.01 \mathrm{~Hz}$ and $50-100 \mathrm{~Hz}$, respectively. The induced torque responses are maximal at contrast frequencies of $0.5-5 \mathrm{~Hz}$.

The results demonstrate that the HSE in Calliphora as well as the torque-generating motor systems in Calliphora and Musca have highly similar contrast frequency characteristics.

\section{Response dependence on the mean luminance of the motion stimulus}

The measurements in this series of experiments were again performed with monocular stimulation of the animals. Direction and velocity of the pattern motion were kept constant $\left(\alpha=0^{\circ}\right.$, $\omega / \lambda=1 \mathrm{~Hz}$ ), and the mean luminance of the grating was varied by means of neutral density filters. 

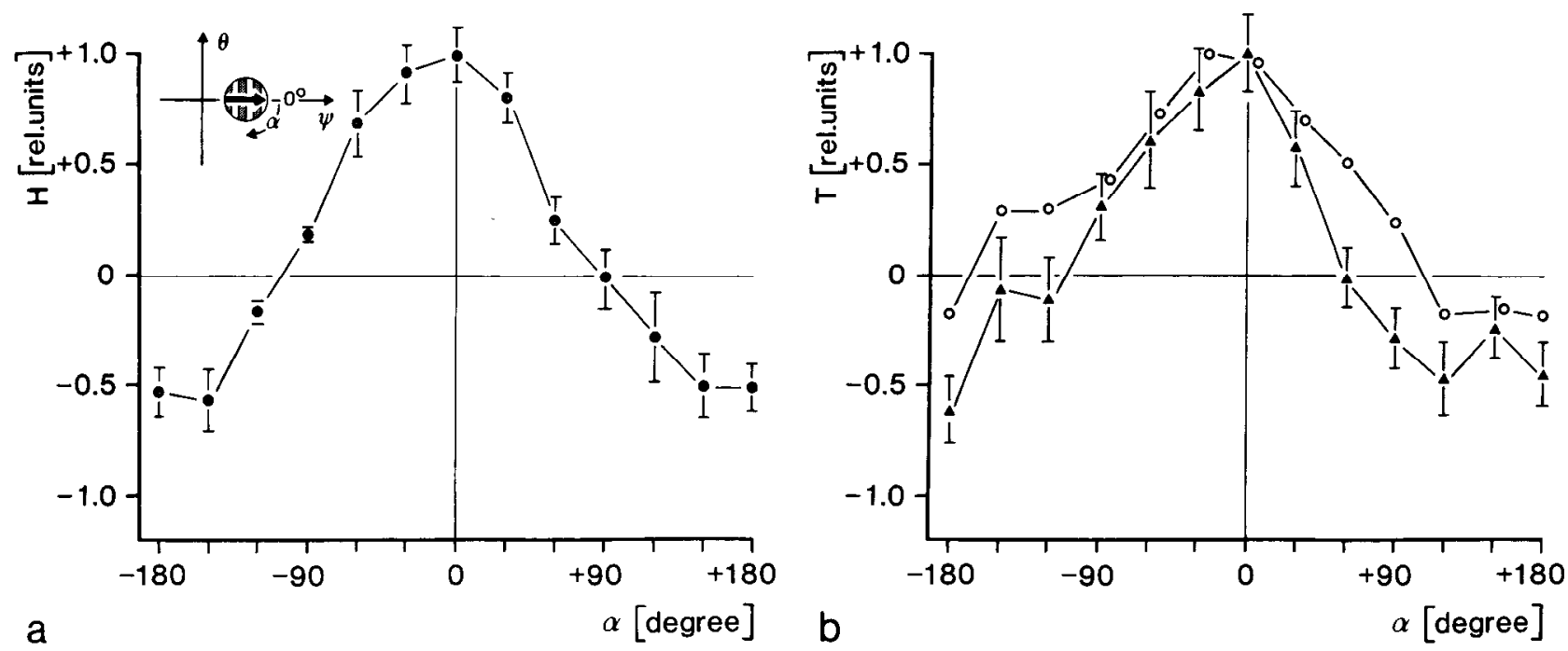

Figure 2. Graded responses of the HSE of Calliphora (a), and flight torque responses $(b)$ of tethered flying Calliphora (O) and Musca ( $\Delta$ ) as functions of the direction $\alpha$ of grating motion. The grating was positioned at $\psi=+40^{\circ}, \theta=0^{\circ}$ (inset) and was moved according to the standard stimulus sequence shown in Figure 1. The direction $\alpha$ of pattern motion was varied in steps of $30^{\circ}$. The other pattern parameters were constant $\left(\omega / \lambda=1 \mathrm{~Hz}, \lambda=13.2^{\circ}, \mathrm{I}=70 \mathrm{~cd} \mathrm{~m}^{-2}, \mathrm{~d}=39.6^{\circ}\right)$. The plotted values are response averages obtained from 15 measurements in HSE of the right lobula plates of 5 flies and from 20 torque measurements in 12 flies of each species. The bars at each point represent SD of the mean. The data were normalized for hetter comparison. The left eyes of the flies were covered with black paint. The curves demonstrate that the cellular responses and the flight torque responses depend similarly on the direction of motion. The HSE is activated preferentially by front to back motion and is inhibited by back to front motion. Positive torque responses indicating intended clockwise turnings of the flies also are elicited by front to back motion. Weak torque responses of opposite sign are induced by motion in the reverse direction.

The electrophysiological results (Fig. $4 a$ ) reveal that the response amplitudes of the HSE strongly depend on the mean luminance $I$ of the stimulus and increase over a range of $2 \log$ units from threshold to saturation. The response threshold lies about 3-4 $\log$ units below the standard luminance $(\log I=0)$ at about 7-70 $\times 10^{-4} \mathrm{~cd} \mathrm{~m}^{-2}$. The results of the respective flight torque measurements in Calliphora (Fig. $4 b$ ) closely fit the electrophysiological data. The data obtained from Musca (Fig. 4b) indicate response saturation at the same luminance level as in Calliphora but differ somewhat with respect to the slope of the response function.

\section{Response dependence on the stimulus position}

Finally, the responses of the HSE and the flight motor system were measured as a function of the stimulus position in the visual field. The pattern was presented in the equatorial plane
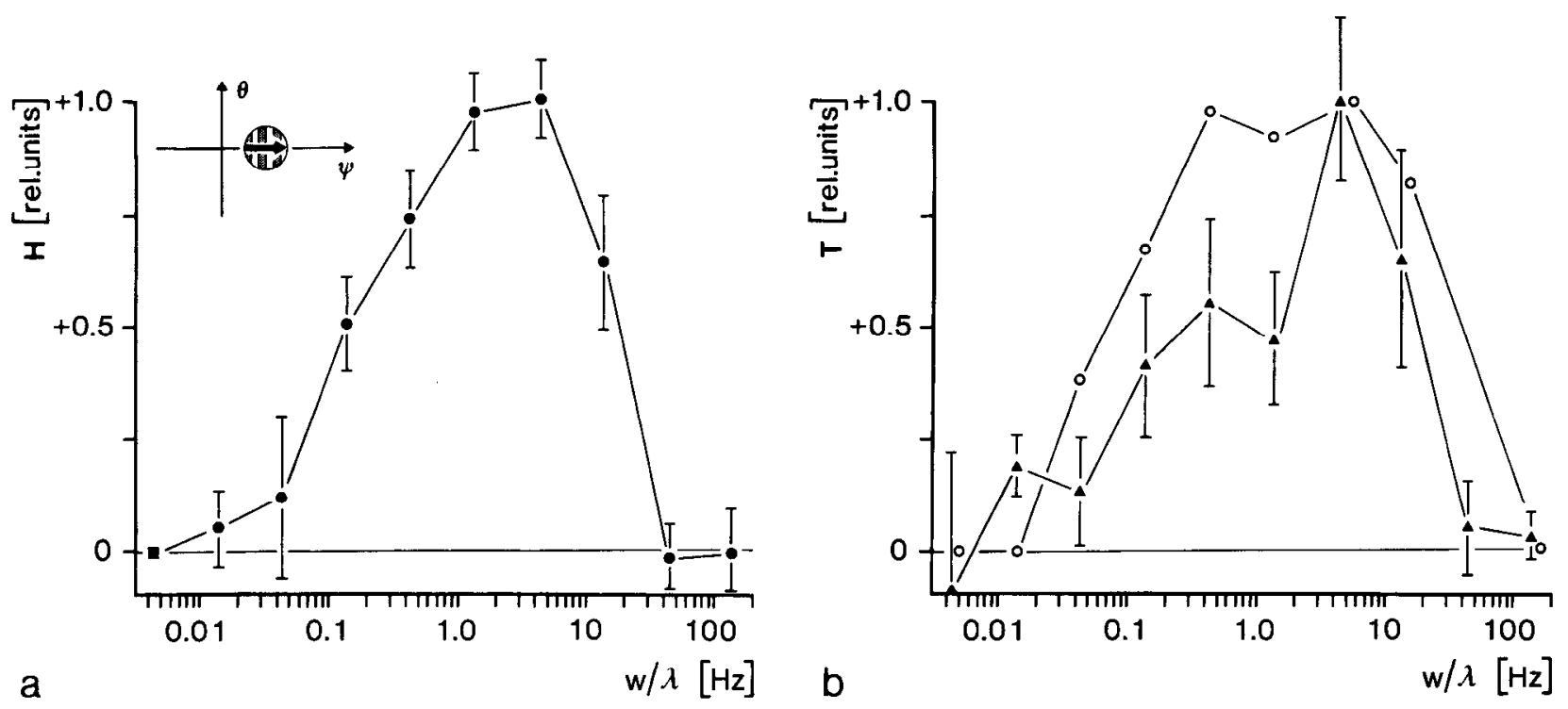

Figure 3. Graded responses of the HSE of Calliphora (a), and flight torque responses $(b)$ of tethered flying Calliphora $(0)$ and $M$ Musca $(\Lambda)$ as function of the contrast frequency $\omega / \lambda$ of grating motion. Contrast frequency (given in $\mathrm{Hz}$ ) of a periodic grating is defined as the ratio between angular velocity (measured in degrees/sec) and spatial wavelength (measured in degrees) of the pattern. Other pattern parameters and data evaluation are described in legend of Figure 2. The cellular and behavioral responses depend strongly and in highly similar manner on the contrast frequency of the stimulus. Peak responses were obtained under stimulation with contrast frequencies of $0.5-5 \mathrm{~Hz}$. 


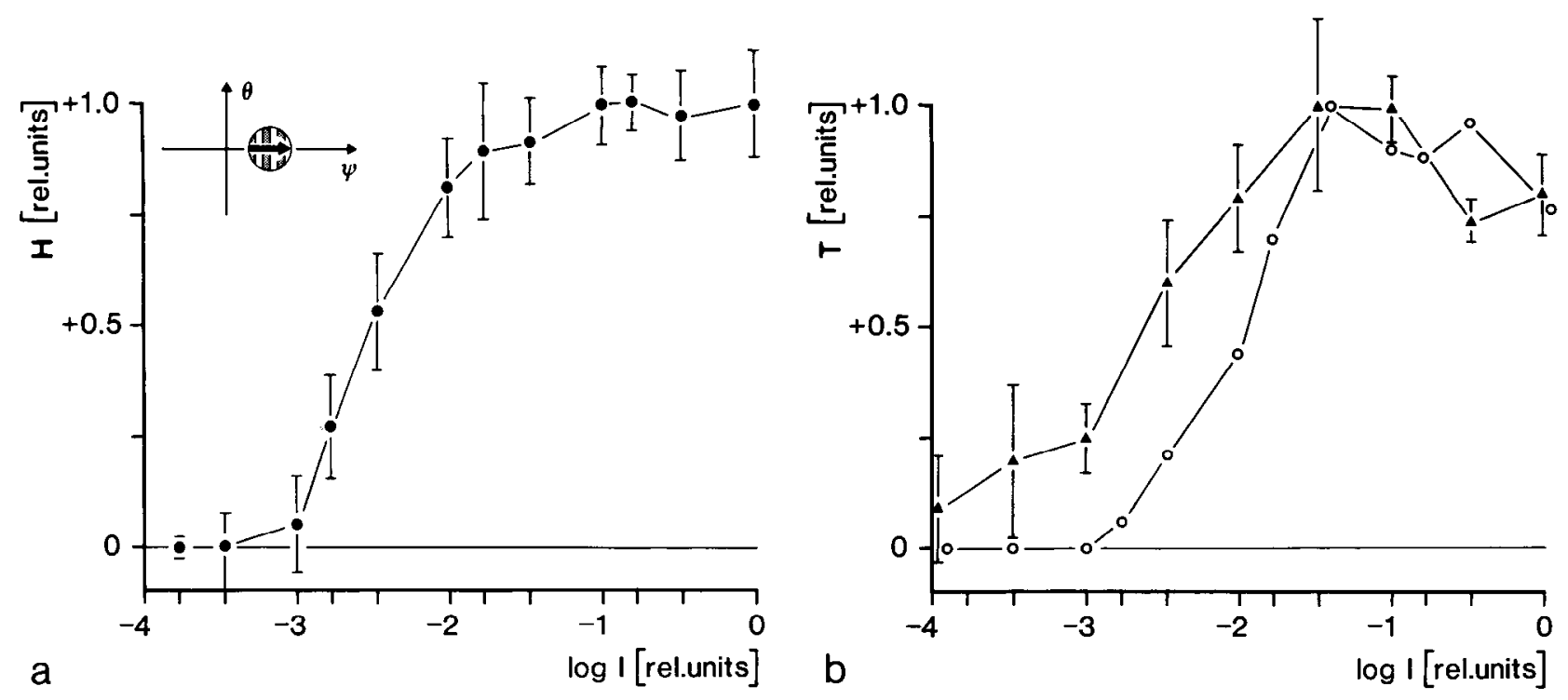

Figure 4. Graded responses of the HSE of Calliphora (a) and flight torque responses (b) of Calliphora (O) and Musca (A) as function of the mean luminance of the moving grating. The standard luminance at $I=0$ equals $70 \mathrm{~cd} \mathrm{~m}^{-2}$ and was varied in steps by means of neutral density filters. Other stimulus parameters and data evaluation as specitied in legend of Figure 2. The curves demonstrate that response threshold and saturation of the cellular and behavioral responses lie at about $3-4 \log$ units and 1-2 log units below standard luminance, respectively.

of the compound eyes and was shifted horizontally in steps of $20^{\circ}$. In each position, the response to the standard stimulation was recorded.

The electrophysiological measurements reveal a characteristic spatial variation in the local sensitivity of the HSE of Calliphora. The moving pattern elicits maximal responses when positioned in front of the animal at $\psi=0-20^{\circ}, v=0^{\circ}$. Stimulation of the lateral part of the right (i.e., ipsilateral) visual field is significantly less effective.

The behavioral measurements in Calliphora and Musca reveal a pronounced difference to the spatial sensitivity distribution of the HSE. In both species, the response maximum is shifted laterally and lies at $\psi=40^{\circ}$ ( Musca) and $\psi=40-60^{\circ}$ (Calliphora). As in the HSE, the response amplitudes decrease for more lateral stimulus positions and decline steeply when the stimulus is moved through the frontal visual field into the contralateral hemisphere.

Thus, in contrast to the results of the previous experiments, the data shown in Figure 5 clearly are not compatible with the notion that, under the present stimulus conditions, the flight torque generation is governed solely by the HSE.

\section{Discussion}

\section{Functional properties of the horizontal system}

In the present study, average responses of the HSE of Calliphora to stimulation with a large, moving grating were evaluated. The experiments complement previous measurements on individual horizontal cells performed under similar conditions (Hausen, $1982 a, b)$. The results of both studies are in close agreement with each other, indicating that response variability between horizontal cells of different animals is low.

The present data clearly show that the HSE responds to horizontal motions and that it is selectively activated by front to back motion. The response, however, also depends on the other stimulus parameters investigated. The cell encodes this particular combination of the parameters of a given motion stimulus rather than simply its direction. In contrast, other factors, such as the size of the stimulus, have only minor influence on the response amplitude (see Fig. 7). The latter property is considered to result from a gain control mechanism, which causes high sensitivity of the cell to small stimuli and prevents response saturation under large-field stimulation (Hausen, 1982b).

As noted, the horizontal system in the lobula plate of the fly comprises 2 further cell types, the HSN and HSS, which scan the dorsal and ventral parts of the ipsilateral visual field, respectively. Although the basic response properties of all three horizontal cells are similar, they show some characteristic functional differences (Hausen, 1982a, b). In the HSN and the HSS, the preferred directions are slightly tilted as compared to that of the HSE. Furthermore, the HSN and HSE, but not the HSS, are additionally activated by back to front motion in the contralateral visual field and must, hence, be regarded as binocular elements encoding horizontal rotations of the environment.

Anatomical studies have revealed that homologous horizontal systems exist in other species of calliphorid flies as well as in Muscidae and Drosophilidae (Pierantoni, 1976; Heisenberg et al., 1978; Eckert, 1981; Fischbach and Heisenberg, 1984; Strausfeld and Bassemir, 1985; Hausen, unpublished observations). Electrophysiological recordings in the calliphorid fly Phaenicia (Eckert, 1981) show that the signals and the general response pattern of the HS cells in this species are almost indistinguishable from those of Calliphora. Detailed physiological studies on the HS systems of Musca and Drosophila are still lacking. Both systems are, however, structurally very similar to the HS system of Calliphora, and preliminary recordings in Musca show that the signals of these cells resemble those of Calliphora (Hausen, unpublished observations). It seems thus justified to assume that the physiological properties of the HS systems in Musca and Drosophila are basically the same as those in Calliphora.

Response properties of the flight torque generating system under large-field stimulation

Flight torque responses to motion have been studied extensively in Musca and Drosophila, but only few investigations have been 

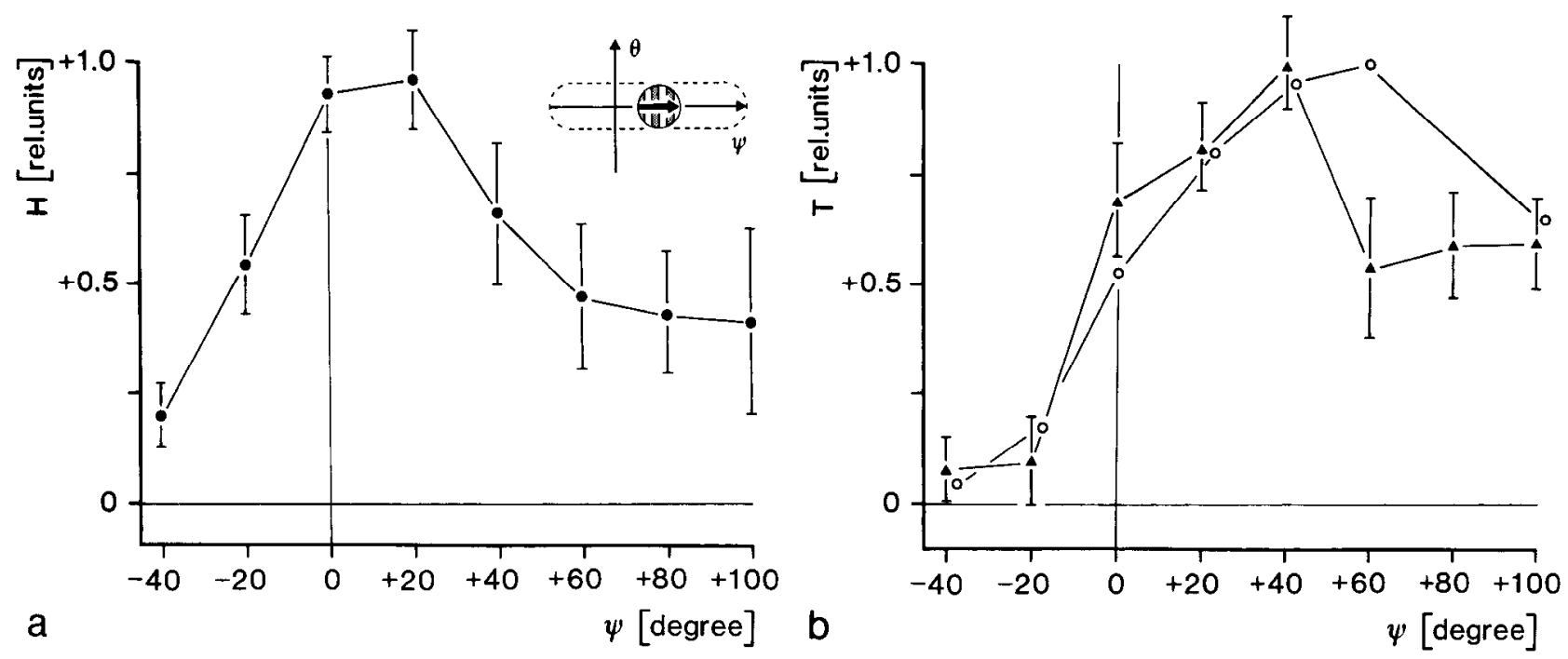

Figure 5. Graded responses of the HSE of Calliphora (a) and flight torque responses $(b)$ of Calliphora $(O)$ and Musca (A) as function of the grating position $\psi$. Other stimulus parameters and data evaluation as specified in legend of Figure 2. The HSE shows a response maximum for grating positions at $\psi=0-20^{\circ}$. The response amplitudes decline steeply when the pattern is moved into the contralateral half of the visual field (left eye covered with black paint) and decrease gradually for pattern positions in the lateral visual field. The behavioral responses differ from the cellular responses with respect to the response maximum, which is found at $\psi=40-60^{\circ}$.

performed in Calliphora (Reichardt et al., 1983; Egelhaaf, 1985a, $\mathrm{b}, \mathrm{c})$. The stimulus conditions differ considerably in the various studies, and a major goal of the present experiments was to gain behavioral data on Musca and Calliphora that allow direct comparisons between the 2 species and that are, in addition, comparable to electrophysiological results.

The present behavioral data reveal, first, that the flight torque responses in both species depend strongly and in a highly similar manner on the stimulus parameters tested. Closer examination of the data reveals response differences between the 2 species of flies in all experiments. These differences, however, are of minor significance and are not interpreted as indications of a different scnsory input organization of the 2 motor systems.

Previous results on Musca concerning the flight torque dependence on the various parameters investigated here agree well with the present data. Peak responses were in all cases found for front to back movement, for contrast frequencies of $1-8 \mathrm{~Hz}$ (Reichardt, 1965; Eckert, 1973; Wehrhahn, 1987), and for stimulus positions at $\psi=20-40^{\circ}$ (Reichardt, 1973; Wehrhahn and Hausen, 1980; Wehrhahn, 1987). The threshold stimulus-luminance of the flight torque response was previously determined at $6 \cdot 10^{-5} \mathrm{~cd} \cdot \mathrm{m}^{-2}$, which is about $1-2$ orders of magnitude below that found here (Eckert, 1971). The latter measurements were, however, performed with a moving panorama stimulating large areas of both eyes, which might account for this difference.

Comparable behavioral measurements also have been performed in Drosophila. Again, front to back motion with a contrast frequency of around $1 \mathrm{~Hz}$ has been found to be the most effective stimulus to elicit flight torque responses (Götz, 1968; Buchner, 1976). Taken together, the available data strongly indicate that the sensory input organization of the motor systems is similar in all three species.

\section{Functional connections between horizontal system and fight motor control}

It is known from studies in Drosophila (Götz, 1968, 1983; Götz et al., 1979) that flight torque is generated mainly by reduction of the beat amplitude of one wing and simultaneous increase of the beat amplitude of the other wing. This differential flight force generation is controlled by steering muscles of the flight motor, which are selectively activated or inhibited by horizontal motion in the visual field (Spüler, 1980; Heide, 1983; Götz, 1983). Since there is anatomical evidence that the horizontal system is connected via interneurons to the thoracic ganglion (Hausen et al., 1981; Strausfeld and Bassemir, 1985; Hausen, unpublished observations), we assume that it is functionally coupled to the motor system such that it inhibits particular ipsilateral steering muscles and activates contralateral ones, causing a simultaneous decrease of the ipsilateral and increase of the contralateral beat amplitudes (see Fig. 6). Hence, monocular front to back motion, which excites the ipsilateral HS system, will lead to the generation of flight torque, turning the flying animal toward the stimulated side. Although back to front motion in front of one eye leads to hyperpolarization of the ipsilateral horizontal cells, which for physiological reasons is unlikely to be transmitted by their output synapses, it can nevertheless elicit turnings in the opposite direction, since the contralateral HSE and HSN are activated by this motion. The observed low efficiency of back to front motion in eliciting torque responses (Figs. 1,2) may be due partly to the particular physiological properties of the horizontal cells. The signals occurring in the HS cells under contralateral stimulation are regular trains of action potentials that differ considerably from the graded responses observed under ipsilateral stimulation. This means that ipsilateral and contralateral inputs originate from different synaptic sitcs. It is thus conceivable that the relative efficiencies of both types of signals in driving the output synapses of the HS cells are different (Hausen, 1982a). Since the transient responses of the HSE and the motor system differ with respect to their time courses (Fig. 1) one has to postulate, in addition, a neuronal low pass filter or leaky integrator in the pathway between HS cells and motoneurons (Reichardt et al., 1983; Egelhaaf, $1985 \mathrm{a}, \mathrm{b}, \mathrm{c})$. In spite of the slight differences between the cellular and the behavioral responses to large-field stimulation, 


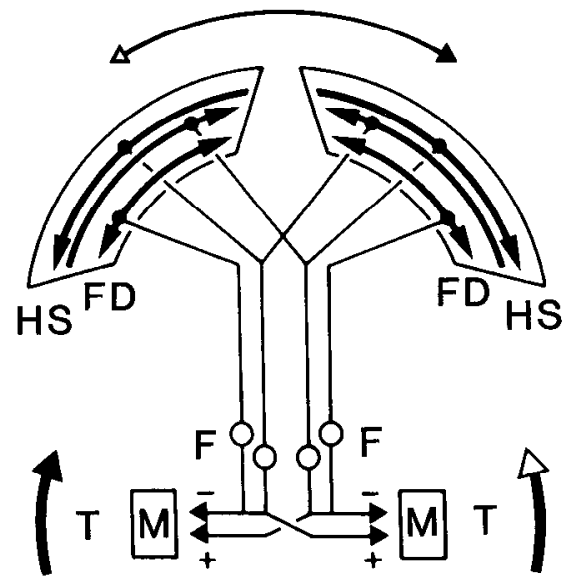

Figure 6. The concept of flight torque control by HS and FD cells. The HS cells of each optic lobe are activated by rotatory global horizontal motion and induce flight torque responses $(T)$ of the flying animal by differential modulation of the ipsilateral and contralateral flight forces produced by the wings. The FD cells are stimulated by local horizontal motion in the ipsilateral visual field and induce flight torque responses toward the stimulus. The signals of the cells are low-pass filtered $(F)$ in the pathway between sensory and motor system.

we regard their general consistency as strong support for the proposed role of the HS cells in behavioral control.

The only evidence indicating the influence of another sensory input to the motor system arises in the present data from the different spatial sensitivities of the HSE and the torque generating system (Fig. 5). Independent evidence in support of an additional torque control system is obtained from flight torque measurements in mutant or lesioned flies.

\section{Flight torque responses of flies with lesions or deficient horizontal systems}

Behavioral measurements in blowflies, the horizontal cells of which were lesioned microsurgically in one optic lobe, have revcalcd that their flight torque responscs to horizontal motion are strongly impaired (Hausen and Wehrhahn, 1983). Under binocular stimulation with moving gratings, the response to front to back motion controlled by the operated optic lobe was found to vanish completely, whereas the response to front to back motion in front of the intact eye remained normal. This result seems to be fully consistent with the proposed connection between HS cells and motor system. It was noticed in this study, however, that monocular stimulation of the operated side still induced torque responses, indicating the existence of an independent cellular system for the control of flight torque, which obviously was not affected by the lesion. These effects are investigated in detail in the second part of this study (Hausen and Wehrhahn, 1990).

Similar observations have been made in houseflies, the horizontal systems of which were unilaterally eliminated by laser ablation in an early larval stage. These animals showed reduced but still significant flight torque responses to monocular stimulation of the ipsilateral eye (Geiger and Nässel, 1981, 1982). The amplitudes of the remaining responses were found to be critically dependent on the size of the stimulus. Whereas the responses to large-field motion were reduced by about $30 \%$, the responses to movement of a single narrow stripe remained un-
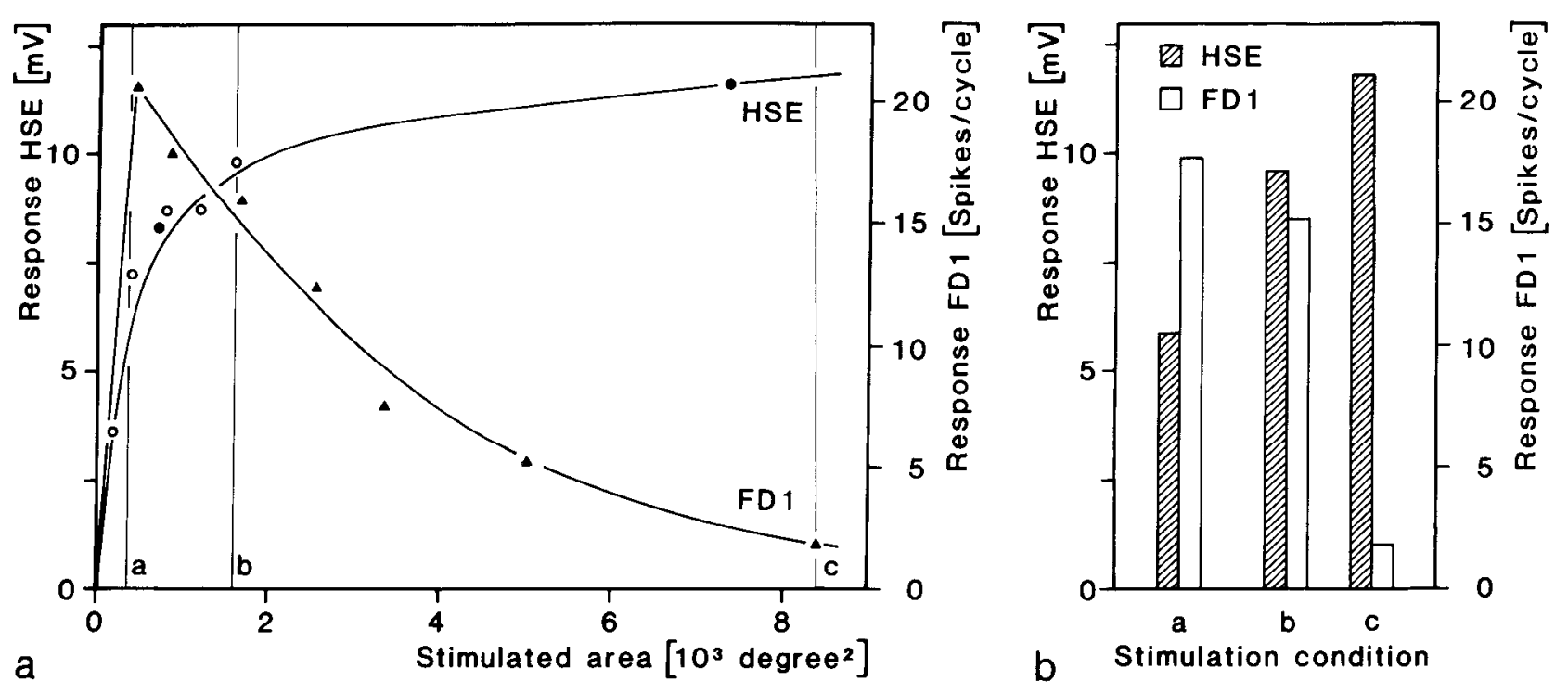

Figure 7. Response dependence of the equatorial horizontal cell HSE and the FD cell FDI of Calliphora on the size of a motion stimulus. The graded responses of the HSE (๑) and the spike activity of the FD1 (A) are plotted as function of the size of a moving panorama segment of constant height $\left(70^{\circ}\right)$ and variable width. The segment was oscillated horizontally around the fly with a frequency of $2.5 \mathrm{~Hz}$ and an amplitude of $\pm 5^{\circ}$, and the cellular responses were recorded during phases of front to back motion (preferred direction of both cells). Vertical lines $a, b, c$ indicate stimulation with a narrow segment (width $5^{\circ}$ ), a medium-sized segment of equal angular size as the grating used in the present study, and a large segment (width $120^{\circ}$ ), respectively. The responses of the 2 cells to these 3 stimuli are compiled in $b$ for direct comparison. $a$, Additional data from an independent measurement on an HSE $(O)$, which was stimulated with front to back motion of a periodic grating, the size of which was increased gradually. The grating was identical to the one used in the present experiments and was displayed in full size at the value marked with the vertical line $b$. It was positioned at $\psi=+30^{\circ}, \theta=0^{\circ}$ and was moved with $\omega / \lambda=1.5 \mathrm{~Hz}$. The curves illustrate the different spatial characteristics of the HSE and the FD1. Whereas the FD1 shows peak responses under stimulation with small patterns, the HSE responds preferentially to stimulation with large patterns. The plot shows, in addition, that the grating used in the present study activates both cells about equally. Data compiled and redrawn from Hausen, 1982b; Reichardt et al., 1983; Egelhaaf, $1985 \mathrm{~b}$. 
altered as compared to the response components controlled by the intact optic lobe.

In Drosophila, the response behavior of mutants deficient of horizontal cells (Heisenberg et al., 1978) was thoroughly investigated. Flight torque responses to a moving panorama were clearly reduced (Blondeau and Heisenberg, 1982). The responses to moving single stripes, however, remained existent in the mutant flies and were recently studied in detail (Bausenwein et al., 1986). From these findings, it was suggested that Drosophila's optomotor flight control is organized by 2 partially parallel subunits, one subunit generating an "object" response the other a "large-field" response.

In conclusion, observations on animals of all 3 species deficient in horizontal cells yield strong evidence in favor of proposed functional role of these cells in flight torque control. There are clear indications that the main significance of the HS cells lies in the processing of large-field motion and that motion of small objects is processed by a separate neural system.

\section{Flight torque responses to stimulation with relative motion of object and background}

Recent studies on the transient response behavior of flies under complex stimulation with a small textured object (stripe) moving in front of a textured panorama gave the first clear evidence that, even in normal flies, motion-induced flight torque responses cannot be explained solely by HS control. Torque generation under this condition relies essentially on the computation of relative motion between object and background (Reichardt et al., 1983), which requires the evaluation of global as well as local motions. The functional properties of the recently described FD cells in Calliphora fit exactly into this concept (Egelhaaf, 1985a, b, c). The FD cells respond selectively to small stimuli moving horizontally within their receptive fields and are binocularly inhibited by horizontal motion of the entire environment. The dynamics of the torque responses of flies to relative motions between an object and the background could be modeled in computer simulations assuming a sensory control network of 2 subsystems equivalent to the HS cells and the FD cells (Fig. 6).

\section{Contributions of HS cells and FD cells to torque control under stimulation with moving gratings}

On the basis of the physiological properties of the HS and the FD cells, a rough assessment of their possible contributions to flight torque control under different stimulus conditions is possible (Fig. 6). Because of their particular spatial integration properties, the HS cells are fully active under stimulation with a moving panorama, whereas the FD cells are inactivated. Hence, the HS cells will dominate the torque control in this situation. In contrast, the FD cells will play the dominant role under stimulation, with a small stripe moving in a contrast-free panorama. The present stimulation with large gratings represents an intermediate situation. As shown in Figure 7, both the HS system and the FD system must be assumed to contribute to torque control under this condition, and the discrepancy between the spatial sensitivity characteristics of the HS cells and the torque generating system found in the present study may well be due to the activity of the FD cells.

To date, 2 FD cells sensitive to front to back motion (FD1, FD4) and 2 further cells sensitive to back to front motion have been described (Egelhaaf, 1985b). In the present context, the former cells are of major interest. Figure 8 shows that the FD1
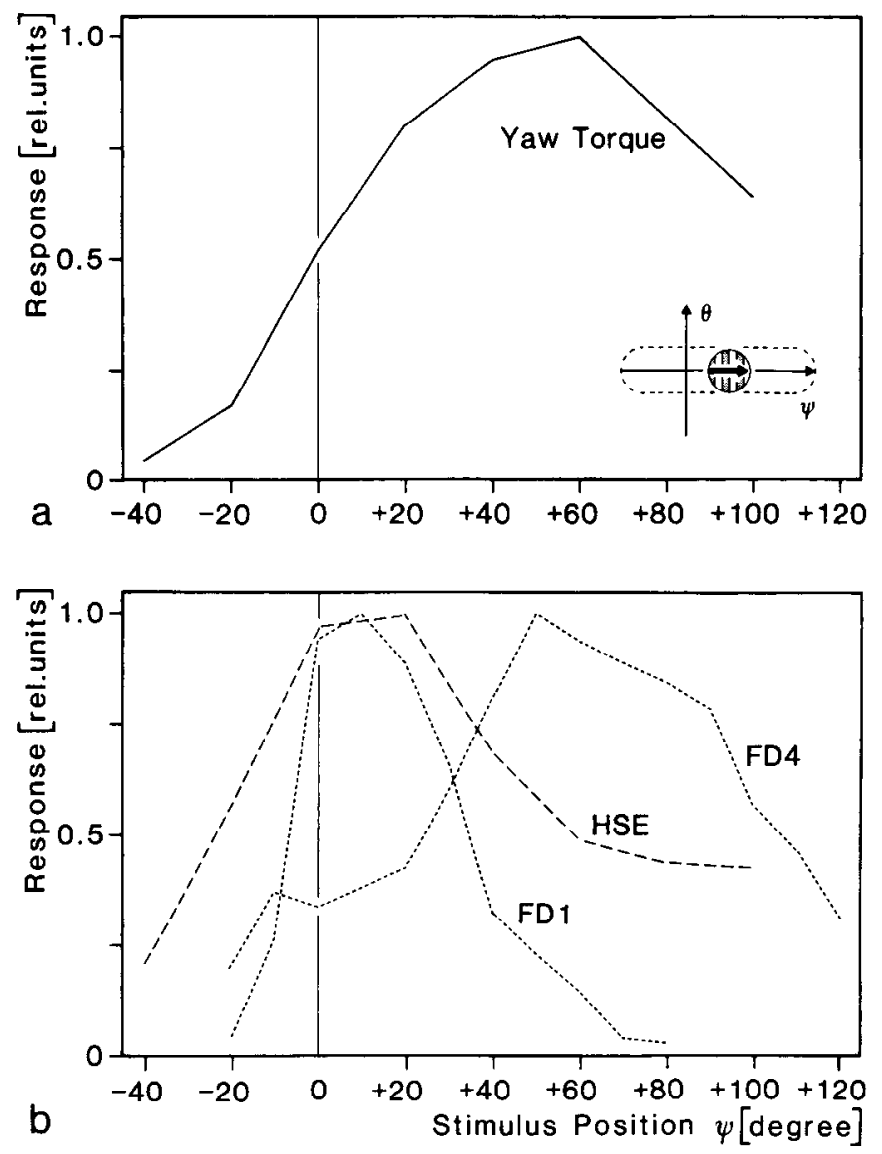

Figure 8. Spatial sensitivity distribution of the sensory system controlling flight torque generation $(a)$ and of the equatorial horizontal cell HSE and the FD cells FD1 and FD4 $(b)$. The behavioral data and the data on the HSE are taken from the present stimulation cxperiments with moving gratings (Fig. 5). The data on the FD cell are redrawn from a previous study (Egelhaaf, 1985b), in which a textured vertical stripe oscillating horizontally in a panorama around the fly was used for stimulation (width of stripe $5^{\circ}$, length of stripe $70^{\circ}$, oscillation frequency 2.5 $\mathrm{Hz}$, oscillation amplitude $+5^{\circ}$ ). The cellular responses were normalized. A comparison of the 2 plots demonstrates that the slope of the curve in $a$ does not fit the spatial sensitivity distribution of the HSE but may well result from superposition of all three cellular response curves if they are weighted accordingly.

of the right lobula plate has a receptive field, which covers the whole dorsoventral extent of the frontal eye region between $\psi$ $=-20^{\circ}$ and $\psi=+80^{\circ}$ and has a sensitivity maximum at $\psi=$ $+10^{\circ}$. The FD4 scans the region between $\psi=-20^{\circ}$ and $\psi=$ $+120^{\circ}$ and reaches its peak sensitivity at $\psi=+50^{\circ}$. The spatial sensitivity characteristics of the HSE and the 2 FD cells and the spatial sensitivity of the torque control system are plotted in Figure 8. This demonstrates that participation of the FD cells in torque control might, in fact, explain the differences between the spatial sensitivity distributions of the HS cells and the motor system. Independent information about the contributions of the sensory subsystems can be gained by lesion experiments in different parts of the brain. The results of such experiments performed on Calliphora will be presented in a subsequent paper.

\section{References}

Bausenwein, B., R. Wolf, and M. Heisenberg (1986) Genetic dissection of optomotor behavior in Drosophila melanogaster. Studies on wildtype and the mutant optomotor-blind ${ }^{\mathrm{H} 3 \mathrm{l}}$. J. Neurogenet. 3: 87-109.

Blondeau, J., and M. Heisenberg (1982) The three-dimensional op- 
tomotor torque system of Drosophila melanogaster. J. Comp. Physiol. 145: 321-329.

Buchner, E. (1976) Elementary movement detectors in an insect visual system. Biol. Cybern. 24: 85-101.

Eckert, H. (1981) The horizontal cells in the lobula plate of the blowfly, Phaenicia sericata. J. Comp. Physiol. 143: 511-526.

Egelhaaf, M. (1985a) On the neuronal basis of figure ground discrimination by relative motion in the visual system of the fly. I: Behavioural constraints imposed on the neuronal network and the role of the optomotor system. Biol. Cybern. 52: 123-140.

Egelhaaf, M. (1985b) On the neuronal basis of figure ground discrimination by relative motion in the visual system of the fly. II: Figuredetection cells, a new class of visual interneurones. Biol. Cybern. 52: 195-208.

Egelhaaf, M. (1985c) On the neuronal basis of figure ground discrimination by relative motion in the visual system of the fly. III: Possible input circuitries and behavioural significance of the FD-cells. Biol. Cybern. 52: 267-280.

Egelhaaf, M., K. Hausen, W. Reichardt, and C. Wehrhahn (1988) Visual course control of flies relies on neuronal computation of object and background motion. TINS 11: 351-358.

Fermi, G., and W. Reichardt (1963) Optomotorische Reaktionen der Fliege Musca domestica. Kybernetik 2: 14-28.

Geiger, G., and D. R. Nässel (1981) Visual orientation behaviour of flies after selective laser beam ablation of interneurons. Nature 293 : 398-399.

Geiger, G., and D. R. Nässel (1982) Visual processing of moving single objects and wide-field patterns in flies: Behavioural analysis after laser-surgical removal of interneurons. Biol. Cybern. 44: 141-149.

Götz, K. G. (1968) Flight control in Drosophila by visual perception of motion. Kybernetik 4: 199-208.

Götz, K. G. (1983) Bewegungssehen und Flugsteuerung bei der Fliege Drosophila. In W. Nachtigall, ed., Biona-Report 2, pp. 21-33, Gustav Fischer, Stuttgart, New York.

Götz, K. G., B. Hengstenberg, and R. Biesinger (1979) Optomotor control of wing beat and body posture in Drosophila. Biol. Cybern. 31: 243-248.

Hausen, K. (1976) Functional characterization and anatomical identification of motion sensitive neurons in the lobula plate of the blowfly Calliphora erythrocephala. Z. Naturforsch. 31c: 629-633.

Hausen, K. (1981) Monocular and binocular computation of motion in the lobula plate of the fly. Verh. Dtsch. Zool. Ges. 1981: 49-70.

Hausen, K. (1982a) Motion sensitive interneurons in the optomotor system of the fly. I. The horizontal cells: Structure and signals. Biol. Cybern. 45: 143-156.

Hausen, K. (1982b) Motion sensitive interneurons in the optomotor system of the fly. II. The horizontal cells: Receptive field organization and response characteristics. Biol. Cybern. 46: 67-79.

Hausen, K. (1984) The lobula-complex of the fly: Structure, function and significance in visual behaviour. In Photoreception and Vision in Invertebrates, M. A. Ali, ed., pp. 523-559, Plenum, New York, London.

Hausen, K., and M. Fgelhaaf (1988) Neural mechanisms of visual course control in insects. In Facets of Vision, D. Stavenga and $\mathbf{R}$ Hardie, eds., Springer-Verlag, Berlin, Heidelberg, New York.

Hausen, K., and R. Hengstenberg (1987) Multimodal convergence of sensory pathways on motoneurons of flight muscles in the fly ( $\mathrm{Cal}$ liphora). Soc. Neurosci. Abstr. 13: 1059.

Hausen, K., and C. Wehrhahn (1983) Microsurgical lesion of horizontal cells changes optomotor yaw responses in the blowfly Calliphora erythrocephala. Proc. R. Soc. Lond. B 219: 211-216.

Hausen, K., and C. Wehrhahn (1990) Neural circuits mediating visual flight control in flies. II. Separation of two control systems by microsurgical brain lesions. J. Neurosci. (in press).

Hausen, K., K. Wolburg-Buchholz, and W. Ribi (1981) The synaptic organization of visual interneurones in the lobula complex of flies. A light and electron microscopical study using silver-intensified cobalt impregnations. Cell Tissue Res. 208: 371-387.

Hausen, K., R. Hengstenberg, and T. Wiegand (1988) Flight control circuits in the nervous system of the fly: Convergence of signal and mechanosensory pathways onto motoneurons of steering muscles. Beitr. 16 Göttinger Neurobiologentagung (in press).

Heide, G. (1983) Neural mechanisms of flight control in Diptera. In Biona-Report 2, W. Nachtigall, ed., pp. 35-52, Gustav Fischer, Stuttgart, New York.

Heisenberg, M., and R. Wolf (1984) Vision in Drosophila, SpringerVerlag, Berlin, Heidelberg, New York.

Heisenberg, M., R. Wonneberger, and R. Wolf (1978) Optomotorblind $^{\text {H31 }}$ a Drosophila mutant of the lobula plate giant neurons. J. Comp. Physiol. 124: 287-296.

Hengstenberg, R., K. Hausen, and B. Hengstenberg (1988) Cobalt pathways from haltere mechanoreceptors to inter- and motoneurons controlling head posture and flight steering in the blowfly Calliphora. Beitr. 16 Göttinger Neurobiologentagung (in press).

Pick, B. (1976) Visual pattern discrimination as an element of the fly's orientation behaviour. Biol. Cybern. 23: 171-180.

Poggio, T., W. Reichardt, and K. Hausen (1981) A neuronal circuitry for relative movement discrimination by the visual system of the fly. Naturwissenschaften 68: 443-446.

Reichardt, W. (1965) Quantum scnsitivity of light receptors in the compound eye of the fly Musca. Cold Spring Harbor Symp. Quant. Biol. 1965: 505-515.

Reichardt, W. (1973) Musterinduzierte Flugorientierung. VerhaltensVersuche an der Fliege Musca domestica. Naturwissenschaften 60 : 122-138.

Reichardt, W. (1986) Processing of optical information by the visual system of the fly. Vision Res. 26: 113-126.

Reichardt, W., and T. Poggio (1976) Visual control of orientation behaviour in the fly. Part I. A quantitative analysis. Q. Rev. Biophys. 9: 311-375

Reichardt, W., T. Poggio, and K. Hausen (1983) Figure-ground discrimination by relative movement in the visual system of the fly. Part II: Towards the neural circuitry. Biol. Cybern. 46[Suppl]: 1-30.

Spüler, M. (1980) Erregende und hemmende Wirkungen visueller Bewegungsreize auf das Flugsteuersystem von Fliegen-Elektrophysiologische und verhaltensphysiologische Untersuchungen an Musca und Calliphora. Doctoral Dissertation, Universität Düsseldorf.

Stewart, W. W. (1978) Functional connections between cells as revealed by dye-coupling with a highly fluorescent naphtalimide tracer. Cell 14: 741-759.

Strausfeld, N. J., and U. K. Bassemir (1985) The organization of giant horizontal-motion-sensitive neurons and their synaptic relationships in the lateral deutocerebrum of Calliphora erythrocephala and $\mathrm{Musca}$ domestica. Cell Tissue Res. 242: 531-550.

Wehrhahn, C. (1986a) Visual guidance of flies during flight. In Comprehensive Insect Physiology, Biochemistry and Pharmacology, Vol. 6, Nervous Systems: Sensory, G. A. Kerkut and L. I. Gilbert, eds., pp. 673-684, Pergamon, Oxford.

Wehrhahn, C. (1986b) Motion sensitive torque responses of the housefly Musca: A quantitative study. Biol. Cybern. 55: 275-280.

Wehrhahı, C., and K. Hausen (1980) How is tracking and fixation accomplished in the nervous system of the fly? A behavioural analysis based on short time stimulation. Biol. Cybern. 38: 179-186. 